\title{
Simulation of Nonlinear Polariton Dynamics in Microcavity wires for Polaritonic Integrated Circuits
}

\author{
G. Slavcheva \\ Physics Dept., University of Bath \\ Bath, BA2 7AY, United Kingdom \\ Email: g.slavcheva@bath.ac.uk
}

\begin{abstract}
M. V. Koleva
\end{abstract}
Physics Dept., University of Oxford

Oxford, OX1 3RH, United Kingdom

Email: mirella.koleva@physics.ox.ac.uk

\author{
A. Pimenov \\ Weierstrass Institute \\ Mohrenstrasse 39,D-10117, Berlin, Germany \\ Email: pimenov@wias-berlin.de
}

\begin{abstract}
We have developed a model of the nonlinear polariton dynamics in realistic strongly-coupled 3D non-planar microcavity wires based on driven-disspative mean-field GrossPitaevskii equations. We find that the typical microcavity nonlinear optical bistability response evolves into multistability upon variation of realistic model parameters and discuss its origin in terms of multiple co-existing transverse cavity modes. Conditions for formation of self-assembled lossless multimode pulses: polariton solitons, and geometry limitations for their stable propagation are identified.
\end{abstract}

\section{INTRODUCTION}

Strong coupling between the cavity mode and the quantum well exciton in a semiconductor microcavity gives rise to mixed light-matter modes, cavity polaritons. Nonlinear optical interactions with microcavity polaritons on a chip are particularly attractive for implementation of polaritonic integrated circuits. This is due to advantages of cavity polaritons over bare photons and excitons alone, such as strong nonlinearities, owing to exciton-exciton interactions, resulting in lower operational powers $\left(\sim f J / \mu m^{2}\right)$, faster switching speeds $(\sim p s)$, tighter localisation and weaker diffraction [1]. Lateral confinement can boost further the exciton-exciton interactions, thereby reducing power consumption. Polaritonic structured media are under active development with arrays of laterally etched microcavity wires currently being fabricated [2]. New tractable theoretical and computational models of the nonlinear dynamics in realistic 3D cavities are therefore needed.

In this work we discuss the polariton nonlinear dynamics in a realsistic non-planar microcavity wire using a scalar drivendissipative mean-field model [3]. We demonstrate spatial multistability, investigate conditions for formation of polariton solitons and identify soliton existence domains and optimal parameters for construction of the building blocks of integrated polariton circuits, implementing soliton logic.

\section{THEORETICAL Model}

We consider a microcavity wire shown in Fig. 1 (a), pumped at an angle $\theta$ along $x$-direction by a linearly polarized $\mathrm{CW}$ wave, polarised along $y$-axis. Solitons propagating along the wire are triggered by a short seed pulse with the same linear

G.S. acknowledges funding through the Leverhulme Trust Research Project Grant No. RPG-2012-481. A. P. acknowledges support from SFB 787 of the DFG. polarization as the $\mathrm{CW}$ pump. The dynamics of the system is described by a scalar mean-field model [3]:

$$
\begin{gathered}
\partial_{t} E-i\left(\partial_{x}^{2}+\partial_{y}^{2}\right) E+\left[\gamma_{c}-i \delta_{c}-i \Delta-i U(y)\right] E= \\
i \Omega_{R}(y) \Psi+E_{p} e^{i \kappa x} \\
\partial_{t} \Psi+\left(\gamma_{e}-i \delta_{e}-i \Delta\right) \Psi+i|\Psi|^{2} \Psi=i \Omega_{R}(y) E .
\end{gathered}
$$

where $E_{p}$ is the normalized pump amplitude, $g$ is the strength of exciton-exciton interaction [4], $\omega_{R}$ is the Rabi frequency in a planar homogeneous cavity, time is measured in units of $T=1 / \omega_{R}$, and $E$ and $\Psi$ are the averages of, respectively, the photon and exciton creation or annihilation operators. The normalisation is such that $\left(\omega_{R} / g\right)|E|^{2}$ and $\left(\omega_{R} / g\right)|\Psi|^{2}$ are the photon and exciton numbers per unit area. $\delta_{e}, \delta_{c}$, and the pump frequency, $\Delta$, are detunings from a reference frequency, $\hbar \omega_{0}=1.55 \mathrm{eV}\left(\lambda_{0}=800 \mathrm{~nm}\right) ; \gamma_{c}$ and $\gamma_{e}$ are cavity and exciton decay rates, assumed equal. The lateral confinement in the cavity plane is described by an effective potential $U(y)$ in the photonic component and a normalized coupling $\Omega_{R}(y)$ :

$$
U(y)=U_{b g}\left[1-e^{-(2 y / w)^{8}}\right], \Omega_{R}(y)=e^{-(2 y / w)^{8}},
$$

where $w$ is the dimensionless wire width and $U_{b g}$ is the background confinement potential.

\section{NUMERICAL RESULTS}

\section{A. Free-polariton dispersion and stationary nonlinear modes}

By setting $E_{p}=0$, neglecting the nonlinear term in Eq. (1)-(2) and using the ansatz $E=A(y) e^{i \kappa x-i \omega t}, \Psi=$ $B(y) e^{i \kappa x-i \omega t}$, the system is recast as an eigenvalue problem. The resulting linear polariton dispersion, $\omega(\kappa)$, at zerodetuning for the fundamental and the next 5 modes of a $3 \mu \mathrm{m}$ wide microwire is shown in Fig. 1 (b). The stationary solutions of the homogeneous nonlinear system Eq. (1)-(2) in the presence of the pump and nonlinearity, are obtained by seeking the solution of the form $\{E, \Psi\}=\left\{A(y) e^{i \kappa x}, B(y) e^{i \kappa x}\right\}$, discretising the $y$ co-ordinate and solving numerically by Newton-Raphson method the resulting nonlinear system. We obtain a bistability nonlinear optical response for $\Delta=-0.1$ and a multi-stable behaviour for $\Delta=0$ (Figs. 1(c,d)) and trace back this spatial multi-stability to the co-existence of multiple transverse cavity modes. We calculate the interval of soliton existence using 2D Newton-Raphson method [3]. 
(a)

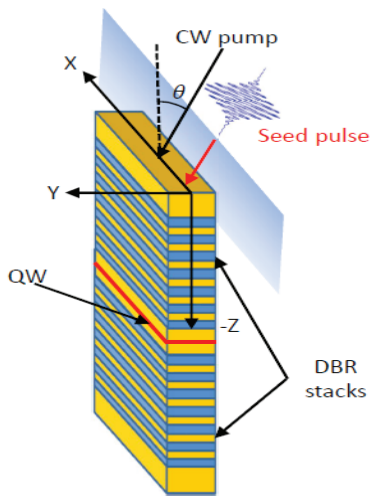

(a)

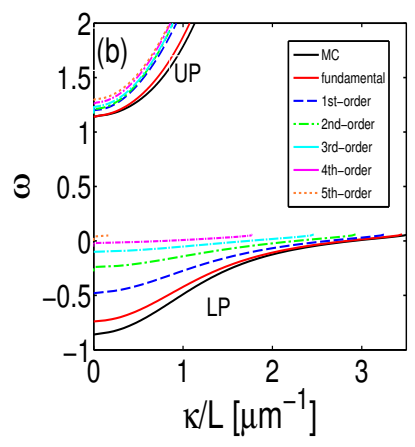

(b)

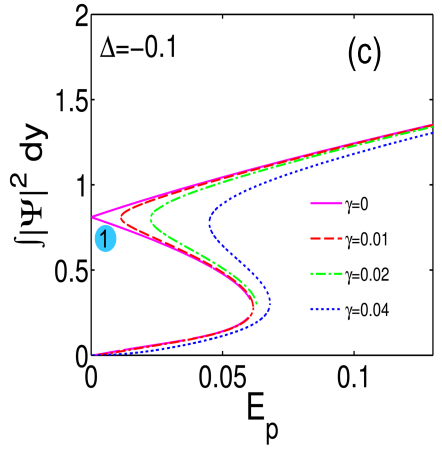

(c)

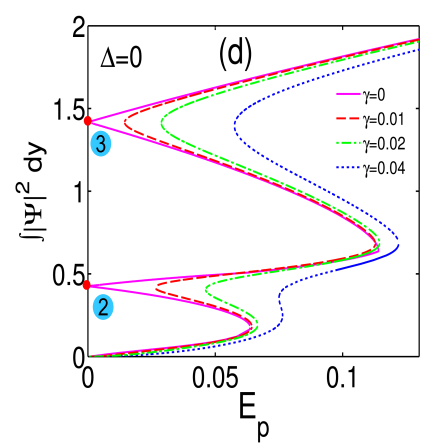

(d)

Fig. 1. (a) Scheme of the microcavity wire structure and the excitation geometry; (b) Linear polariton dispersion for the fundamental and 5 higher-order modes at $\Delta=0$, wire width $w=3 \mu \mathrm{m}$ (lower (LP) and upper (UP) polariton branches). The planar microcavity dispersion is displayed with solid black lines; (c) Bistability curve for $\Delta=-0.1$ and (d) Multistability curve for $\Delta=0$ of the integrated $\Psi$-field power vs $E_{p}$ for $\gamma=0,0.01,0.02,0.04 ; \theta=20^{\circ}$.

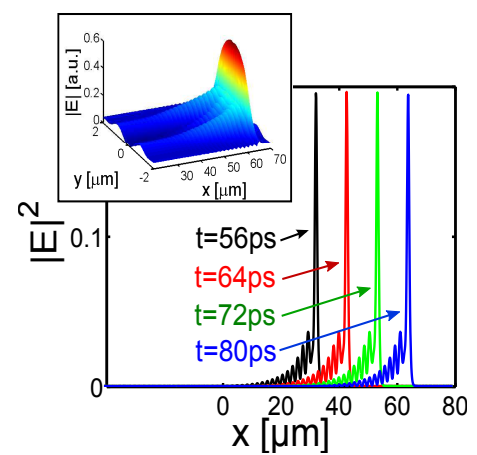

(a)

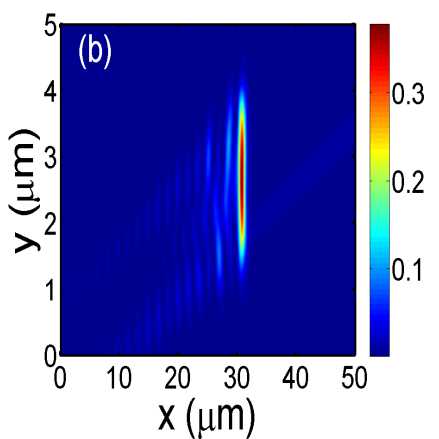

(b)

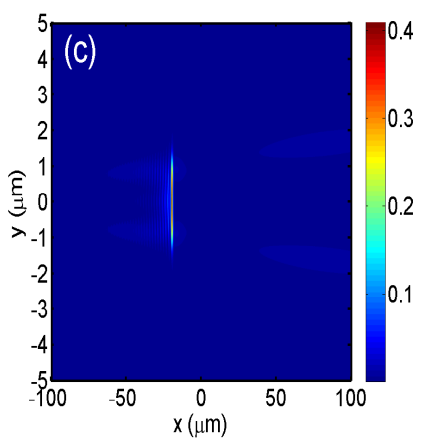

(c)

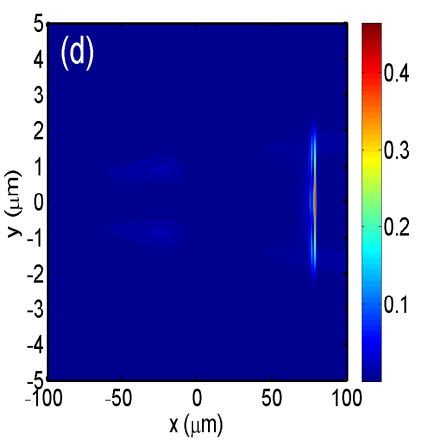

(d)

Fig. 2. (a) Snapshot at $t=80$ ps of the intensity of a single-soliton at $\Delta=0$ and $\gamma=0.04$ for pump amplitude $E p=0.075$ and a cross-sections at four different times: $t=56,64,72$ and $80 \mathrm{ps}$ along the middle of the microcavity wire; (b) Top view of a soliton at $t=24$ ps in a tilted wire ( $\alpha=5^{\circ}$ with respect to the $x$ axis); (c,d) time evolution of a soliton propagating in a tapered wire: snapshots at $t=48$ ps and $t=120$ ps.

\section{B. Dynamical modelling of soliton formation and propagation in straight, tilted and tapered wires}

We choose the pump amplitude within the soliton existence domain and initialise the system with the stable mode from the lowest branch (Fig.1(d)). The soliton formation is triggered at time $t=0$ by a short ( $\tau=2 \mathrm{ps)}$ Gaussian seed pulse, collinear with the pump. The system Eqs. (1) and (2) is solved in the time domain by the split-step method and the soliton solution is displayed in Fig. 2 for straight (a), tilted (b) and tapered (c,d) microcavity wires. By 2D Newton we found a maximum tilt angle $\alpha_{\max } \approx 12 \pm 2^{\circ}$ at $E_{p}=0.075$, which imposes limitations in designing splitters, routers and other functional components of the future polaritonic integrated circuits.

\section{CONCLUSiON}

We have developed a model of the polariton nonlinear dynamics in non-planar microcavity wires, based on drivendissipative, 2D mean-field Gross-Pitaevskii equations. For a realistic microcavity wire, we found that the conventional for planar microcavities bistability evolves into complex multi- stability and discussed its origin in detail [3]. In contrast to the single-mode polariton solitons in planar microcavities, polariton solitons in microcavity wires exhibit a complex multi-mode structure. The multi-mode solitons deserve special attention, since they are capable of propagating further along an imperfect microcavity than their single-mode counterparts. We have developed a modal expansion method [5] to investigate the nonlinear mechanisms behind localisation, and attempt to find a means to leverage the inter-modal interactions for the development of polaritonic integrated circuits.

\section{REFERENCES}

[1] A. Baas, J. P. Karr, H. Eleuch, and E. Giacobino, "Optical bistability in semiconductor microcavities,” Phys. Rev. A, vol.69, p. 023809, 2004

[2] E. Wertz et al.,"Spontaneous formation and optical manipulation of extended polariton condensates", Nat. Phys., vol. 6, pp. 860-864, 2010

[3] G. Slavcheva, A. V. Gorbach, A. Pimenov, A. G. Vladimirov and D. V. Skryabin, "Multi-stability and polariton solitons in microcavity wires,", Opt. Lett. vol. 40, pp. 1787-1790, 2015

[4] M. Sich et al., "Observation of bright polariton solitons in a semiconductor cavity,” Nature Photonics, vol. 6, pp. 50-55, 2012

[5] G. Slavcheva, A. V. Gorbach, and A. Pimenov, "Coupled spatial multimode solitons in microcavity wires,” Phys. Rev. B vol. 94, 245432, 2016 